\title{
Photoinduced Reorientation and Polarization Holography of Photoreactive Polymer Liquid Crystals with Bistolane Side Groups
}

\author{
Nobuhiro Kawatsuki,* Ayumi Yamashita, Mizuho Kondo, Hiroshi Ono a) and Akira Emoto ${ }^{\text {a) }}$ \\ Department of Materials Science and Chemistry, Graduate School of Engineering, \\ University of Hyogo, 2167 Shosha, Himeji 671-2280, Japan \\ a) Department of Electrical Engineering, Nagaoka University of Technology, \\ 1603-1 Kamitomioka, Nagaoka 940-2188, Japan \\ kawatuki@eng.u-hyogo.ac.jp
}

\begin{abstract}
New photo-cross-linkable liquid crystalline polymethacrylate with 4-methoxybistolane side groups was synthesized and its thermally enhanced photoinduced molecular reorientation behavior was investigated using a linearly polarized (LP) UV light. Since the bistolane mesogenic side groups were axis-selectively photoreacted under irradiating with LPUV light, annealing procedure generated the thermally enhanced molecular reorientation parallel to the polarization (E) of LP-365 nm light. The axis-selectively photoreacted bistolane moieties acted as the photo-cross-linked anchor to reorient non-photoreacted side groups along them, which direction was parallel to $\mathbf{E}$. The maximum generated in-plane order parameter and birefringence were 0.43 and 0.18 , respectively. The influences of degree of the photoreaction and the annealing temperature on the molecular reorientation behavior were investigated in detail. Furthermore, polarization holography using $325 \mathrm{~nm} \mathrm{He}-\mathrm{Cd}$ laser was carried out to fabricate pure polarization gratings with various polarization of the light source.
\end{abstract}

Key words: tolane, photoalignment, photo-orientation, polymer liquid crystal, birefringence

\section{Introduction}

Photoinduced molecular reorientation has received much attention because of its practical applications such as optical and holographic memory devices, birefringent optical films, and the photoalignment for liquid crystal display devices. Two main categories of photoreactive materials have been investigated to attain the molecular reorientation of thin films so far: One is the azobenzene-containing polymer, which is based on the axis-selective $E-Z-E$ photoisomerization. [1-3] Another is based on the axis-selective photo-cross-linking of the liquid crystalline polymer (PLCP) comprised of cinnamate or cinnamic acid mesogenic side groups. [4] For the azobenzene system, rewritable molecular orientation is achieved although it is not transparent in the visible region. Thermally stable molecular reoriented film can be obtained by the use of PLCPs with cinnamate side groups, and it is applicable to the optical films for the display devices because cinnamate is transparent in the visible region. [5]

We have carried out a systematic study on the photoinduced molecular orientation of PLCPs with cinnamate-containing mesogenic groups. [6-9] The axis-selectively photoreacted mesogenic groups acted as photo-cross-linked anchor to reorient whole the mesogenic groups parallel to them based on the thermally induced self-organization. Besides the cinnamate groups, it is known that a tolane moiety undergoes the axis-selective photoreaction. Ichimura's group reported the alignment control of low-molecular-weight liquid crystals on a polymeric film with tolane side groups that were axis-selectively photoreacted by LPUV light. [9] 
However, the molecular reorientation of the tolane groups was not observed. There are few studies on the photoinduced reorientation of polymeric films containing tolane moiety.

In general, the main purpose of the use of tolane groups is to improve the birefringence of the material. [10] Ikeda's group investigated that the photoinduced reorientation of azobenzene-containing polymeric films attached with tolane moiety. [11-13] Copolymerization of the tolane containing mesogenic groups also investigated to improve the photoinduced birefringence. $[14,15]$ We also studied the thermally enhanced photoinduced orientation of tolane-containing polymers with cinnamate end groups, where the improvement of the generated birefringence was attained compared to that based on the biphenyl mesogenic groups. [16] In these cases, tolane moieties did not act as the photo-cross-linkable groups because of the low photoreactivity compared to that of azobenzene and cinnamate groups. We reported a thermally enhanced molecular reorientation of a polymethacrylate film with 4-methoxytolane side groups based on the axis-selective photoreaction of the tolane moieties, but the efficiency of the photoinduced reorientation was low and the generated birefringence was less than 0.05. [16] Large photoinduced molecular reorientation of PLCP films based on the axis-selective photoreaction of the tolane moiety has not been achieved so far.

In this paper, to utilize the axis-selective photoreaction of the tolane moiety, a new polymethacrylate film with 4-methoxybistolane side groups (P1 in Scheme 1) was synthesized. The thermally enhanced photoinduced reorientation behavior of P1 films was investigated, and the influences of the degree of the photoreaction of the tolane groups and the annealing temperature on the molecular reorientation behavior were explored using a polarization UV spectroscopy. Finally, polarization holography using this PLCP film was fabricated using a $325 \mathrm{~nm}$ He-Cd laser with various polarizations.

\section{Experimental}

\subsection{Materials}

Scheme 1 outlines the synthesis of a methacrylate monomer with 4-bistolane side groups 6. Synthetic procedure is described elsewhere. The chemical structures of the monomers (1-6) were confirmed by ${ }^{1} \mathrm{H}-\mathrm{nmr}$ and IR spectroscopes. PLCP P1 was synthesized by a radical solution polymerization from 6 in THF at $53{ }^{\circ} \mathrm{C}$. Number and weight average of molecular weight of the synthesized polymer were $2.1 \times 10^{4}$ $\mathrm{g} / \mathrm{mol}$ and $3.2 \times 10^{4} \mathrm{~g} / \mathrm{mol}$, respectively. P1 exhibited nematic liquid crystalline phase between 96 and $202^{\circ} \mathrm{C}$.

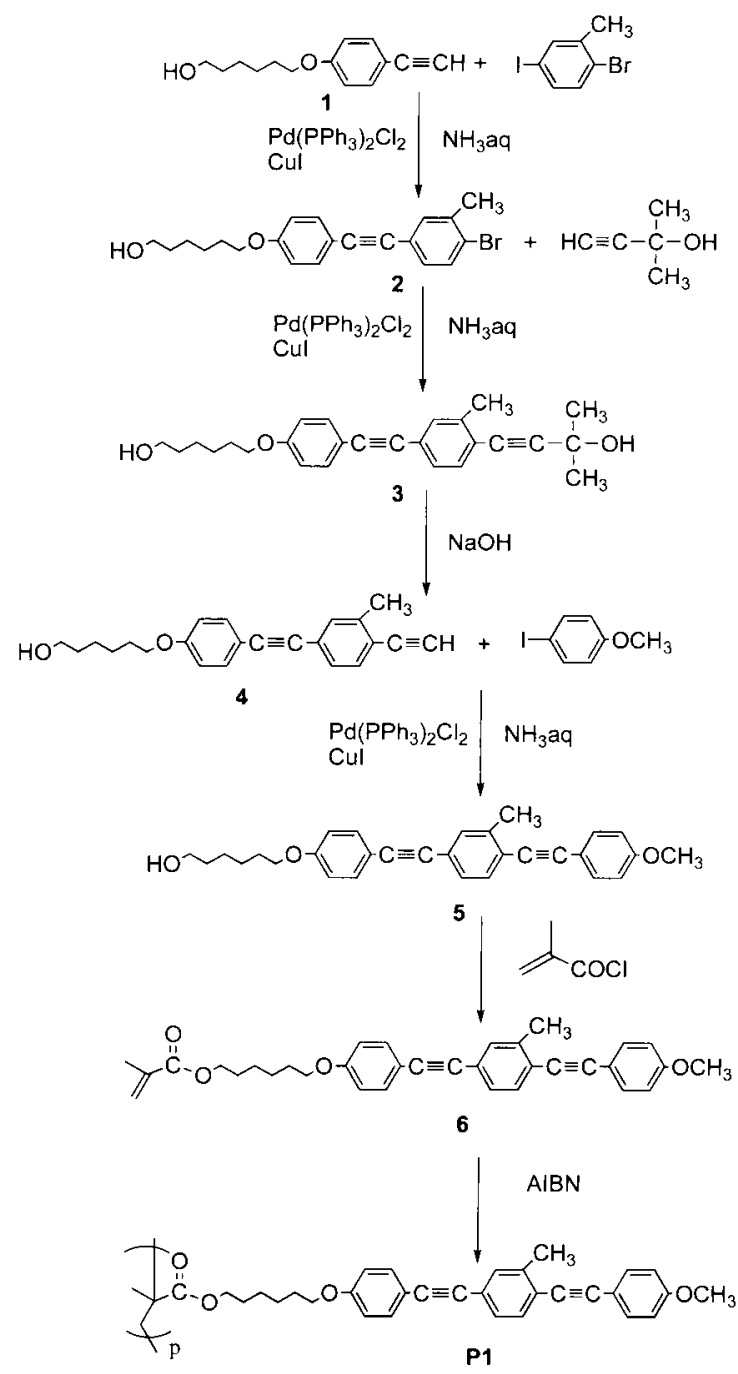

Scheme 1 Synthetic route for $\mathbf{P 1}$

\subsection{Photoirradiation and characterization}

A thin P1 film was prepared by a spin-coating method on a quartz substrate from methylene chloride solution, resulting in a $0.2 \mu \mathrm{m}$-thick film. The film was irradiated by LP-365 nm light from a 250W ultra high-pressure Hg-UV lamp (25 $\mathrm{mW} / \mathrm{cm}^{2}$ at $365 \mathrm{~nm}$ ) equipped with Glan-Tayor polarization prisms and band-pass filter at $365 \mathrm{~nm}$. The degree of the photoreaction was evaluated by monitoring the absorbance at $320 \mathrm{~nm}$ in UV-vis spectroscopy.

For molecular reorientation, the exposed film 
was annealed at elevated temperatures for $10 \mathrm{~min}$. The photoinduced and thermally enhanced optical anisotropies of the film were measured by polarizing optical microscopy (POM) and polarization UV-vis spectroscopy.

The in-plane order parameter, $S$, is expressed in the form of equation 1 ;

$$
S=\frac{\mathrm{A}_{\|}-\mathrm{A}_{\perp}}{\mathrm{A}_{\text {(large) }}+2 \mathrm{~A}_{\text {(small) }}}
$$

where $A_{\|}$and $A_{\perp}$ are the absorbances parallel and perpendicular to $\mathbf{E}$, respectively. $A_{\text {(large) }}$ is taken to be the larger value of $A_{\|}$and $A_{\perp}$, and $A_{(\text {small) }}$ is taken to be the smaller one. $S$ is calculated at $320 \mathrm{~nm}$.

\subsection{Polarization holography}

Polarization holographic grating was recorded with orthogonal linear (OL) exposure using two beams with $s$ - and $p$-polarized components. [17] Orthogonal circular (OC) exposure consisting of two opposing circular polarization ( $\mathrm{R}-$ and $\mathrm{L}-$ ) beams was also carried out. A $\mathrm{He}-\mathrm{Cd}$ laser (Kinmon Koha, IK3501R-G-S) at a wavelength of $325 \mathrm{~nm}(20 \mathrm{~mW}, 4 \mathrm{~mm} \phi)$ was used as the light source. The optical set-up for polarization holography is illustrated in Fig. 1. After the exposure, the sample film was annealed at $155^{\circ} \mathrm{C}$ to generate a molecular reorientation. The grating constant $(\sqcup)$ was set to $11 \mu \mathrm{m}$ by adjusting the angle between two light beams. The holographic gratings were analyzed using a probe beam emitted from a He-Ne laser at a wavelength of $632.8 \mathrm{~nm}$. For first order diffraction, the diffraction efficiency was measured using a photo-detector, and the polarization states were evaluated using a polarizer placed behind the sample plane.

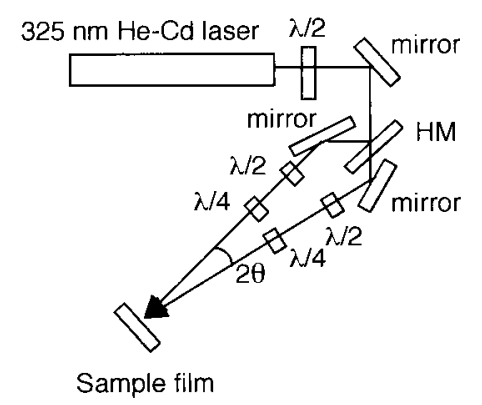

Fig. 1. Experimental setup for polarization holography

\section{Results and discussion}

3.1. Axis-selective photoreaction of a $\mathbf{P 1}$ film

Fig. 2 shows change in the absorption spectrum of a P1 film when the film was exposed to LP-365 $\mathrm{nm}$ light. It reveals that the absorption band around $300-370 \mathrm{~nm}$ decreases, indicating the photoreaction of the tolane moieties. Furthermore, the inset figure shows the photoinduced optical anisotropy $\left(\Delta A=A_{\|}-A_{\perp}\right)$ as a function of the exposure energy. It reveals that a negative $\Delta \mathrm{A}$ is generated and increased as the photoreaction proceeds, but $\Delta \mathrm{A}$ decreased when the exposure energy was greater than $50 \mathrm{Jcm}^{-2}$. Additionally, the exposed films were insoluble in organic solvents. These are due to the axis-selective photo-cross-linking reaction of the bistolane side groups. The axis-selective photoreaction of the monotolane groups was reported previously. $[9,16]$ The decrease in the $\Delta \mathrm{A}$ at higher exposure doses is because that the photoreaction in the perpendicular direction to $\mathbf{E}$ proceeded similar to the case of the axis-selective photoreaction of cinnamate side groups. [4]

The photoreaction of the bistolane moiety was confirmed by FT-IR spectrum. Fig. 3 shows change in the FT-IR spectrum of a P1 film exposed to LP-365 nm light. The absorption band at $2200 \mathrm{~cm}^{-1}$ decreased when the photoreaction proceeded. This means the photoreaction of the tolane moieties. However, it is unknown which triple C-C bonds in the bistolane moiety preferentially photoreacts at present.

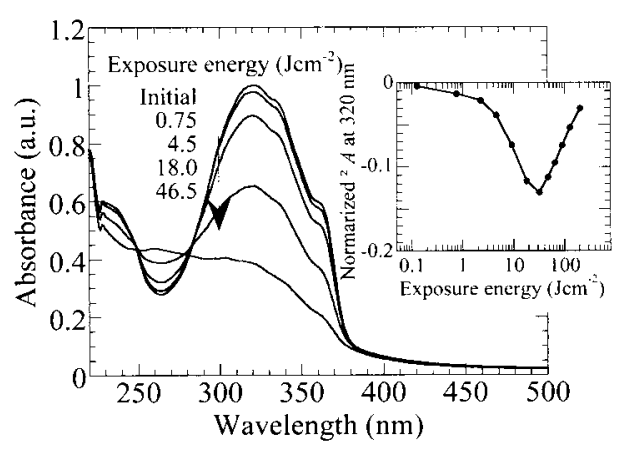

Fig. 2. Change in the absorption spectrum of $\mathbf{P} 1$ film. Inset shows change in the photoinduced optical anisotropy.

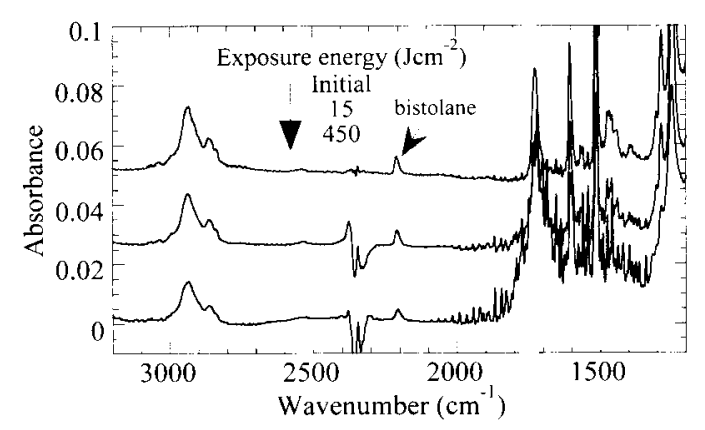

Fig. 3. Change in the FT-IR spectrum of P1 film.

3.2. Thermal enhancement of the photoinduced reorientation 
In case of the PLCP with cinnamate-containing mesogenic side groups, a small photoinduced optical anisotropy is reversely enhanced when the exposed film is annealed in the LC temperature range of the material. $[7,8,16]$ This is because that the axis-selectively photoreacted cinnamate side groups acted as the photo-cross-linked anchor for the thermally induced self-organization. For the polymethacrylate with monotolane side groups, the thermal amplification of the photoinduced $\Delta \mathrm{A}$ was very small. [16] In contrast, sufficient molecular reorientation was achieved for the PLCP with bistolane side groups $\mathbf{P 1}$.

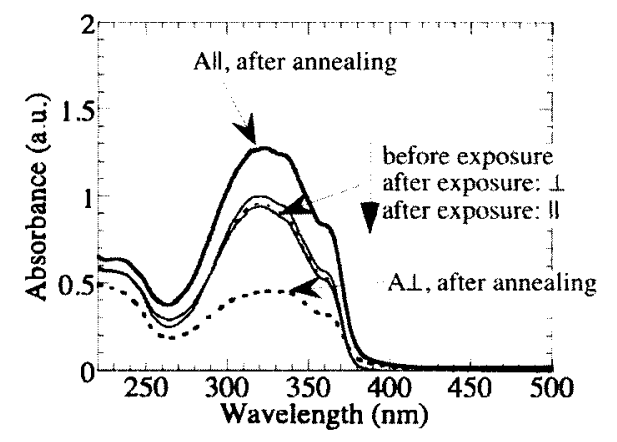

Fig. 4. Change in the polarized absorption spectrum of a P1 film before and after irradiating with LPUV light, and after subsequent annealing.

Fig. 4 plots change in the UV polarization spectra of a $\mathbf{P 1}$ film before and after exposing to LP-365 nm light, and subsequent annealing at $155^{\circ} \mathrm{C}$ for $10 \mathrm{~min}$, when the maximum molecular reorientation was obtained. The degree of the photoreaction (DP) was $4.5 \%$. The irradiating with LP-365 nm light cased a small negative $\Delta \mathrm{A}$. After the annealing, reversion and the thermal enhancement of the photoinduced $\Delta \mathrm{A}$ was observed. The in-plane order was changed from -0.02 to +0.43 . This enhanced $S$ value is somewhat smaller than that for PLCPs with cinnamate side groups, but larger than with monotolane side groups. $[7,16]$ The axis-selectively photo-cross-linked mesogenic side groups, which act as the anchor for the molecular reorientation, induced the thermally enhanced molecular reorientation parallel to E. [4] Namely, the axis-selectively photo-cross-linked bistolane side groups acted as the photo-cross-linked anchor parallel to $\mathbf{E}$, which is similar to the case of the photoinduced molecular reorientation of cinnamate-containing PLCP films. [7] Additionally, the generated birefringence was
0.18 at $633 \mathrm{~nm}$. The large generated birefringence is due to the large inherent birefringence of the bistolane moiety. Large photoinduced birefringence in azobenzene-containing polymer with bistolane groups has been also investigated. [18]

3.3. Influence of exposure doses

The DP affected the efficiency of the thermal enhancement of the molecular reorientation because the photo-cross-linking influences on the self-organization of the mesogenic side groups. Fig. 5 plots thermally enhanced $S$ values of $\mathbf{P 1}$ film as a function of DP when the irradiated film is annealed at $155^{\circ} \mathrm{C}$. Large in-plane orientation $(S>0.4)$ was obtained when DP was $4-5 \%$. The required DP for the efficient molecular reorientation for the PLCPs with biphenyl mesogenic groups attached with cinnamate end groups was $10-20 \%$. [7] Lower required DP for the efficient molecular reorientation was observed for PLCPs with tolane mesogenic groups attached with cinnamate end groups. [16] These results indicate that small amount of photo-cross-linked anchors can generate the thermally-induced self organization of the (bis)tolane mesogenic groups.

3.4. Influence of annealing temperature

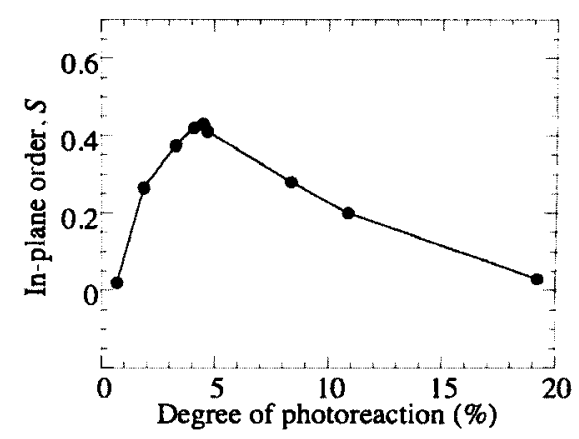

Fig. 5. Thermally enhanced $S$ values of $\mathbf{P 1}$ films as a function of degree of the photoreaction. Films were annealed at $155^{\circ} \mathrm{C}$ for $10 \mathrm{~min}$ after the photoreaction.

Influence of the annealing temperature on the thermal enhancement was evaluated using irradiated films with $4.5 \%$ of DP. Fig. 6 plots the results. When the annealing temperature was between $140{ }^{\circ} \mathrm{C}$ and $185{ }^{\circ} \mathrm{C}$, molecular reorientation occurred. This temperature range is consistent with the LC temperature range of P1. This is similar to other PLCPs that exhibit thermally enhanced photoinduced molecular reorientation. $[3,7,16]$ 


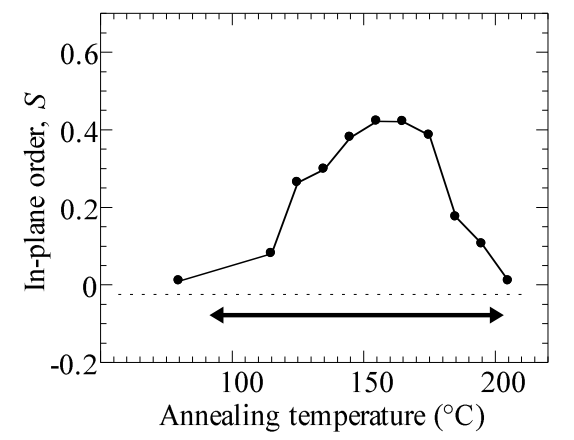

Fig. 6. Thermally enhanced $S$ values of $\mathbf{P 1}$ films as a function of annealing temperature. DP of films was $4.5 \%$. Arrow indicates LC temperature range of $\mathbf{P 1}$.

\subsection{Polarization holography}

We previously reported that photoinduced molecular reorientation of PLCP films containing cinnamate-mesogenic side groups can be applicable to pure polarization hologram using 325 $\mathrm{nm}$ He-Cd laser. [17] However, polarization holography based on the axis-selective photoreaction of tolane moiety, which also absorbs $325 \mathrm{~nm}$ light, has not been explored. Polarization holography of $\mathbf{P 1}$ films was carried out using two beams of $s$ - and $p$-polarizations (OL beams) or oppositely circularly-polarizations (OC beams). In these conditions, polarization state of the interference beams is modulated but the interferometric beam intensity is constant as illustrated in Fig. 7.

For example, Fig. 8a shows POM photograph of fabricated gratings with OL beams. The exposure time was $5 \mathrm{~s}$ and the exposed film was annealed at $200{ }^{\circ} \mathrm{C}$ for $10 \mathrm{~min}$. It reveals that a bright area appears every $5.5 \mu \mathrm{m}$. This is a half pitch of the grating period. AFM observation revealed that the surface relief structure was less than $5 \mathrm{~nm}$. These results indicated that the periodic molecular reorientation constructed the pure polarization gratings. Similar formation of the polarization grating was investigated using other

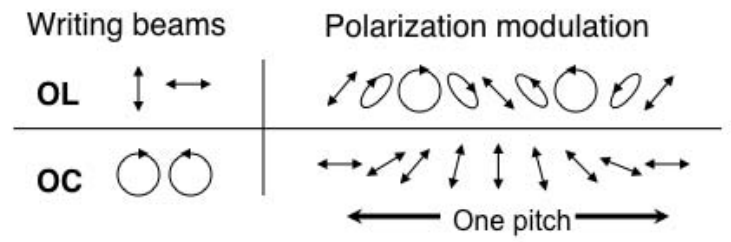

Fig. 7. Polarization states of two writing beams for polarization holography.

PLCP films with cinnamate mesogenic side groups. [17] A polarization grating fabricated with OC beams also exhibited the oriented structure shown in Fig. 7. (a)

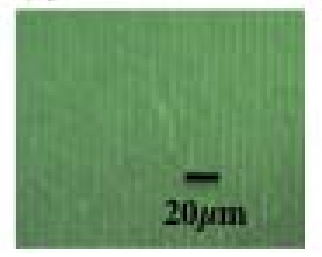

(b)

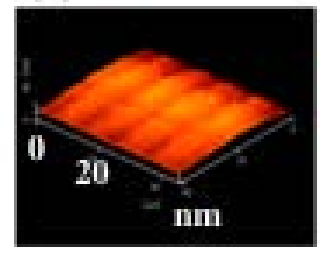

Fig. 8. (a) POM and (b) AFM photographs of polarization grating fabricated by OL beams.

One of the features of the polarization gratings is the conversion of the polarization on of the diffracted beams. [17] For the grating fabricated with OL beams, the polarization of the first order diffracted beams was rotated by $90^{\circ}$, when the linearly polarized probe beam was used. In contrast, for the grating fabricated by $\mathrm{OC}$ beams, incident linearly polarized light was converted to the circularly polarized \pm first order diffraction beams. These experimental results are consistent with the theoretical predictions for pure polarization gratings. [17]

\section{Conclusion}

Thermally enhanced molecular reorientation of a new PLCP containing 4-methoxybistolane side groups was investigated. Due to the axis selective photo reaction of the tolane groups, the irradiating with LP-365 nm light induced negative optical anisotropy of the film, and the subsequent annealing process generated molecular reorientation parallel to $\mathbf{E}$ of the LP light. The generated in-plane order parameter was 0.43 and the birefringence was 0.18 . For an application of this PLCP, fabrication of pure polarization gratings was demonstrated and the resultant gratings exhibited the similar optical properties as previously fabricated using other PLCPs. The tolane photoreactive moiety can be useful for the axis-selective photoactive groups for the photoinduced molecular reorientation of PLCP system. Synthesis of other PLCPs with tolane photoreactive groups attached with other type of mesogenic core is underway.

\section{Acknowledgements}

This his work was partially supported by a grant-in-aid of Scientific Research in Priority Areas "New Frontiers in Photochromism (No. 471)" from the Ministry of Education, Culture, Sports, Science and Technology (MEXT), and a grant-in-aid of 
Scientific Research B (No. 21350129) and S (No. 21225006) from the Japan Society for the Promotion of Science.

\section{References}

1. K. Ichimura, Chem. Rev., 100 (2000) 1847, M. O'Neill and S. M. Kelly, J. Phys. D: Appl. Phys., 33 (2000) R67.

2. T. Ikeda, J. Mater. Chem., 13 (2003) 2037.

3. A. Natansohn, P. Rochon, Chem. Rev. 102 (2002) 4139.

4. N. Kawatsuki and N, Ono H. "Organic Electronics and Photonics", Vol. 2; H. S. Nalwa Ed. American Sci. Publishers, Stevenson Ranch, CA, (2007) 301.

5. N. Kawatsuki, T. Kawakami and T. Yamamoto, Adv. Mater, 13 (2001) 1337, K. Ichimura, Y. Akita, H. Akiyama, K. Kudo and Y. Hayashi, Macromolecules, 30 (1997) 903.

6. N. Kawatsuki, H. Ono, H. Takatsuka, T. Yamamoto and O. Sangen, Macromolecules, 30 (1997) 6680, N. Kawatsuki, H. Takatsuka, T. Yamamoto and H. Ono, Jpn. J. Appl. Phys., 34 (1997) 6464.

7. N. Kawatsuki, K. Goto, T. Kawakami and T. Yamamoto, Macromolecules, 35 (2002) 706.

8. N. Kawatsuki, H. Takatsuka, T. Yamamoto and
O. Sangen, J. Polym. Sci., Part A: Polym. Chem., 36 (1998) 1521.

9. M. Obi, S. Morino and K. Ichimura, Chem. Mater, 11, (1999) 1293.

10. F. Ye, A. Orita, J. Yaruba, T, Hamada T and J. Otera, Chem. Lett., 33, (2004) 528.

11. K. Okano, A. Shishido and T. Ikeda, Macromolecules, 39 (2006) 145.

12. K. Okano, A. Shishido and T. Ikeda, $A d v$ Mater., 18, (2006) 523.

13. S. Yoneyama, T. Yamamoto, O. Tsutsumi, A. Kanazawa, T. Shiono and T. Ikeda Macromolecules, 35 (2002) 8751.

14. M. Ishiguro, D. Sato, A. Shishido and T. Ikeda, Langmuir, 23 (2007) 332.

15. Y. Kitani, C. Kitamura, A. Yoneda and N. Kawatsuki, Mol. Cryst. Liq. Cryst., 443 (2005) 181.

16. N. Kawatsuki, A. Yamashita, Y. Fujii, C. Kitamura and A. Yoneda, Macromolecules, 41 (2008) 9715.

17. N. Kawatsuki, T. Hasegawa, H. Ono and T. Tamoto, Adv. Mater, 15 (2003) 991, H. Ono, A. Emoto, N. Kawatsuki and T. Hasegawa, Appl. Phys. Lett., 82 (2003) 1359.

18. K. Okano, O. Tsutsumi, A. Shishido, and T. Ikeda, J. Am. Chem. Soc., 128 (2006) 15368. 